\title{
Lead Site V1
}

National Cancer Institute

\section{Source}

National Cancer Institute. Lead Site V1. NCI Thesaurus. Code C90412.

A unipolar electrocardiogram lead site; the electrode is placed at the fourth intercostal space on the anterior chest wall (between ribs 4 and 5) to the right of the sternal border. In small animals, it is placed at the right fifth intercostal space near the sternum. 